\title{
STABILITY CHARACTERISTICS OF COMPRESSIBLE BINARY PLANAR JETS
}

\author{
M. M. Vargas \\ and M. T. Mendonca ${ }^{\mathrm{b}}$ \\ ${ }^{\mathrm{a}}$ Instituto de Pesquisas Espaciais \\ Laboratório Associado de Combustão e \\ Propulsão, \\ Cachoeira Paulista, SP, 12630-000, Brasil \\ myl_vargas@hotmail.com \\ ${ }^{\mathrm{b}}$ Instituto de Aeronáutica e Espaço \\ São José dos Campos, SP, 12228-904, Brazil \\ marciomtm@fab.mil.br \\ ABSTRACT \\ The present work investigates the stability of compressible binary planar \\ jets. Different from a homogeneous jet, where a single chemical species is \\ present, the binary jet may have strong density gradients due to the choice \\ of the chemical species considered in each stream. The goal is to identify \\ the possible instability modes for simple and co-flowing jets and \\ investigate the effect of density gradients on the flow structure, growth \\ rates, unstable frequency range and disturbance phase speed for each mode. \\ The effect of species concentration on free shear layer stability has been \\ reported previously in the literature, but detailed comparisons between \\ stability modes and characteristics for a range of density ratios typical of \\ oxygen and hydrogen mixtures as well as the identification of inner and \\ outer sinuous and varicose modes are new. Linear stability theory is used to \\ determine the stability characteristics of the different configurations. For the \\ co-flowing jet four different modes are found, the inner and outer shear \\ layers both have sinuous and varicose modes. Both for the sinuous and \\ varicose modes the simple jet is more unstable when the fluid with the \\ highest density is at the inner jet, with amplification rates twice as high as \\ the lowest density ratio considered, but the range of unstable frequencies \\ can be four times lower. The sinuous mode is less dispersive than the \\ varicose and the disturbance speeds may vary by one order of magnitude \\ with density ratio. For co-flowing jets the external mode is up to seven \\ times more unstable, but this is due to the choice of the velocity ratio \\ considered. For the inner mode the density gradient has a stabilizing effect \\ regardless of which species is at the center. The co-flowing jet is more \\ dispersive, except for the varicose inner mode. The variation of phase speed \\ with density gradient is not as strong as in the simple jet. The ratio of larges \\ Received: June 04, 2019 \\ Revised: July 14, 2019 \\ Accepted:May 13, 2020 \\ to lower phase speeds are of the order of 2 for the co-flowing jet and 4 for \\ the simple jet. \\ Keywords: compressible jets; hydrodynamic stability; numerical simulation
}

\section{NOMENCLATURE}

cp dimensionless phase speed

$G \quad$ Gropengiesser variable

Ma Mach number

$\mathrm{M}_{\mathrm{c}} \quad$ convective Mach number

$\hat{p} \quad$ pressure eigenfuction

$\mathrm{R}$ real part of a complex variable

$R \quad$ thermodynamic gas constant

$R_{1 / 2} \quad$ dimensionless jet half diameter

$R_{\text {ratio }}$ gas constant ratio

$t \quad$ dimensionless time

$u \quad$ dimensionless streamwise velocity component

$\mathrm{v}$ dimensionless normal velocity component

$x \quad$ dimensionless streamwise direction coordinate

y dimensionless normal direction coordinate

z dimensionless spanwise direction coordinate

$T$ dimensionless temperature

\section{Greek symbols}

$\alpha \quad$ complex wavenumber

$\alpha_{r} \quad$ streamwise wavenumber $\alpha_{i} \quad$ streamwise growth rate

$\beta \quad$ spanwise wavenumber

$\beta \gamma \quad \gamma_{1} / \gamma_{2}$

$\beta \rho \quad$ density ratio

$\beta_{U} \quad$ velocity ratio

$\gamma \quad$ specific heat ratio

$\chi \quad$ Gropengiesser variable

$\omega \quad$ angular frequency

$\varphi$ dependent variable

$\hat{\phi} \quad$ complex amplitude eigenfunction

$\rho \quad$ dimensioless density

$\rho_{\text {ratio density ratio }}$

$\theta \quad$ momentum thickness

$\zeta$ normal direction distance

\section{INTRODUCTION}

Hypersonic vehicles require high speed combustion in order to reduce pressure losses due to chock waves, but compressibility has a strong stabilizing effect that hinders the proper mixing of reactants in the combustion chamber. The present investigation addresses the problem of stability of 
compressible jets considering different chemical species in the flow, representing streams of different concentrations of oxidizer and fuel.

Jackson has done extensive work on the stability of compressible, uniform and binary flows. In an early work Jackson and Grosch (1989) studied the spatial stability of a compressible mixing layer. The methodology and conclusions apply to other free shear layers in compressible flow in terms of the effect of Mach number, temperature ratios and direction of the most unstable modes. They classify the disturbances as subsonic, fast mode, slow mode and supersonic, depending on the phase speed and on the Mach number. The details of the slow and fast modes were latter complemented by the investigation of Zhuang et al. (1990). Jackson and Grosch (1989) also show that the boundary conditions away from the shear layer support both exponentially decaying disturbances and radiating outgoing waves for the fast, slow and supersonic modes. One year later, Jackson and Grosch (1989) investigated the stability of compressible mixing layers and identified the transition from convective to absolute instability. They defined an expression for the convective Mach number for flows with different chemical species as the relevant compressibility parameter for binary mixing layers. The convective Mach number for binary mixing layers is defined as

$$
M_{c}=\operatorname{Ma}\left(1-\beta_{u}\right) /\left(1+\sqrt{\beta_{\gamma} / \beta_{\rho}}\right)
$$

Where $M a$ is the Mach number of the fast stream, $\beta_{u}, \beta \gamma$ and $\beta_{\rho}$ are the velocity, specific heat ratio and density ratios between the fast and slow streams.

The stability of rectangular jets has been studied by Tam and Thies (1993) using a vortex sheet model. They found different instability modes, where the dominant mode is called the central mode since the largest fluctuation amplitude is located at the centre of the jet. Corner instability modes are relevant close to the nozzle jet exit and are responsible for smearing the jet into a more rounded profile downstream.

Kennedy et al. (1998) investigated the effect of temperature variations in compressible jets using boundary layer similar solutions for the base flow profiles. They found that a hot jet into a cold ambient have growth rates ten times greater than a jet in isothermal conditions and that the range of unstable frequencies is also greater. On the other hand a cold jet is more stable than the isothermal jet. The results are related to density gradients and have a greater effect on growth rate than compressibility effects.

Weder (2012) investigated the stability and acoustics of compressible subsonic planar jets using linear stability theory. Imposing canonical velocity profiles typical of turbulent flows they were able to recover instability and acoustic modes that are relevant for noise generation and propagation. The analysis of disturbance energy balance presented suggests the main mechanisms associated with disturbance growth to be due to the production term in the Reynolds-Orr energy equation, while growth associated with thermal effects are minor.

Regarding the study of binary mixtures in free shear layers, a number of publications may be found. Kennedy and Gatski (1994) presented results for laminar shear layers composed of nitrogen and hydrogen. They solve the self-similar boundary layer equations for compressible, binary mixing layers, considering variable properties such that Prandtl, Chapman-Rubesin and Lewis numbers vary continuously across the layer. The results show that variable properties have a significant effect on the velocity, temperature, density and mass fraction profiles. As a result, the vorticity structure decouples from the density profile for large density ratios. The variable properties results have strong implications for flow stability as shown later in different investigations.

In a more extensive investigation than the one presented by Kennedy and Gatski. (1994), Kozusko et al. (1996a), investigated the laminar flow structure of a binary, compressible mixing layer for thirty different combinations of gas mixtures. The gas properties are considered variable and dependent on temperature and mass fraction distributions, such that Lewis and Prandtl numbers vary considerably across the layer. The resulting velocity, density, temperature and mass fraction profiles are very different from profiles obtained for constant nondimensional numbers approximation and are not necessarily symmetric. The results also depend on the denser gas been on the fast or slow stream. These results show the importance of correctly modeling the base flow for stability analysis. In the same issue, Kozusko et al. (1996b) studied the stability of compressible, binary mixing layers. The results are presented in terms of the ratio between the molecular mass on the two streams, since it has a significant effect on the phase speeds, growth rates and range of unstable frequencies. Nevertheless, the normalized growth rate for a given convective Mach number is not strongly dependent on the gas composition.

The temporal linear stability of compressible binary shear layers with large density ratios was investigated by Fedioun and Lardjane (2005). The stabilizing effect of compressibility for convective Mach numbers above 0.6 was reported and showed to be independent of the density ratio. As compressibility effects increase, the most amplified mode becomes three-dimensional. For binary flows the most amplified mode, the corresponding wavelength and the wave speed, depend on the species considered, temperature distribution and free stream velocities, and not only on the convective Mach number.

Salemi and Mendonca (2008) investigated the stability of compressible mixing layers when different chemical species are present on the two 
streams. They studied the effect of compressibility and mass fraction stratification on the mixing layer stability. Following the work of Salemi and Mendonca, Mendonca (2014) investigated the stability of mixing layers modified by jets and wakes. Both the effect of more complex flow structures and the effect of large density gradients due to different chemical species in each stream were assessed. The base flow velocities, temperature and mass fraction distributions were given by the solution of boundary layer equations in an intent to have more realistic conditions than the ones given by canonical profiles. The results show that the combined effect of different species and distortion of the mixing layer by jets and wakes have a strong effect on the stability characteristics.

The results by Mendonca (2014) were further investigated by Manco et al. (2015) to study compressible mixing layers, jets and wakes composed of different chemical species in the different stream. They used direct numerical simulations to analyze the flow structures resulting from the stability of complex mixing layers modified by wakes and jets. Again, realistic base flow variables were evaluated using results from boundary layer simulations, and velocity, temperature and mass fraction profiles are significantly different from canonical profiles used in other studies. The results show that the more complex base flows enhance instability, which is responsible for stronger mixing between reactants. The fastest growing disturbances for the different conditions considered were determined.

The stability of compressible binary planar jets was investigated by Rogensky et al. (2017) using direct numerical simulations. The laminar viscous flow is obtained from Navier-Stokes simulations and stability results are obtained from a high order of accuracy Euler solver. They considered two different cases, a hydrogen jet in an oxygen environment and an oxygen jet on a hydrogen environment. They found that, for the conditions investigated, the sinuous mode when an oxygen jet is considered, is the most unstable. The flow structures are presented through the velocity and vorticity fields in the nonlinear regime, for the most unstable sinuous and varicose cases.

Following the methodology presented by Rogenski et al. (2017), Lacerda et al. (2018) performed additional direct numerical simulations of planar jets and mixing layers in the compressible flow regime. Results were compared to linear stability theory for a range of Mach numbers, showing the stabilizing effect of compressibility. The nonlinear regime was also studied through the analysis of the vorticity field, showing the structure of the sinuous and varicose instability development.

The literature review presented above is far from exhaustive, but presents the main topics that are relevant for the investigation in the present research.
As seen above, there are a considerable amount of work on the stability of mixing layers and jets in compressible homogeneous flows. Considering binary free shear flows, there was an intense interest in the nineteen nineties and early two thousand, when the main features of binary free shear layers have been identified. The detailed analysis of the stability properties dependence on density stratification presented here will provide additional information related to the mixing of reactants in combustion systems.

The objective of the present investigation is to identify the supported instability modes in binary coflowing planar jets and study how the amplification rate, phase speed and range of unstable frequencies vary with the choice of density ratio between the different species. The following questions are addressed. Do the inner and outer shear layers in a co-flowing jet support sinuous and varicose modes? If so, how do they compare in terms of growth rate, dispersion and range of unstable frequencies? How does the simple jet and co-flowing jet stability characteristics vary for a range of density ratios between $1: 16$ to $1: 1 / 16$, typical of hydrogen and oxygen mixtures? Which configuration is the most unstable in terms of growth rate and unstable frequency range? How does the disturbance phase speed varie with frequency for different density ratios, in other words, how does disturbance dispersion varies with density ratio?

The paper is organized as follows, after this introduction, the mathematical model based on inviscid linear stability equations are presented. Then, results are presented for simple planar binary jets and then for co-flowing planar binary jets. Conclusions are presented in the last section.

\section{MATHEMATICAL MODEL}

The flow configuration consists of two streams issuing from a nozzle into a quiescent ambient. The fast stream of a given chemical species is surrounded by a slower stream of a different chemical species, as presented in Fig. 1.

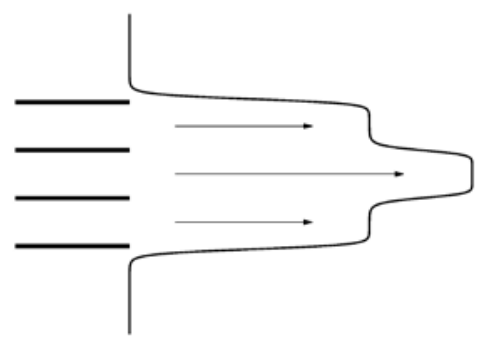

Figure 1. Planar, two-dimensional base flow configuration with fast inner stream and symmetric slower outer stream.

The problem is formulated as a stability problem by reducing the compressible Euler 
equations to the compressible version of the Rayleigh stability equation, starting with the non-dimensional Euler equations in Cartesian coordinates for threedimensional compressible flows of a binary mixture of two different gases, with reference values given by the stream at the center.

In order to derive the stability equation the instantaneous flow variables are decomposed in a steady base flow and a disturbance,

$$
\phi(x, y, z, t)=\bar{\phi}(y)+\phi^{\prime}(x, y, z, t)
$$

where the base flow has been assumed parallel, $x, y$, and $z$ are the streamwise, normal and spanwise coordinates and $t$ is the time.

Solution for the resulting set of equations for the disturbances may be sought by normal modes, assuming

$$
\phi^{\prime}=\{\hat{\phi}(\mathrm{y}) \exp [\mathrm{i}(\alpha \mathrm{x}+\beta \mathrm{z}-\omega \mathrm{t})]\}_{\mathrm{R}}
$$

where $\hat{\phi}(\mathrm{y})$ is a complex amplitude, $\alpha=\alpha_{r}+i \alpha_{i}$ where $\alpha_{r}$ is the streamwise wavenumber and $\alpha_{i}$ is the streamwise growth rate, $\beta$ is the spanwise wavenumber and $\omega$ is the angular frequency.

From the disturbance equations and from the normal mode solution, applying the change of variables proposed by Gropemgiesser (1970), after some mathematical manipulation one can arrive at the following equations:

$$
\begin{gathered}
\chi=\frac{\mathrm{i} \alpha \mathrm{p}}{\gamma \mathrm{Ma}^{2} v}, \\
\frac{d \chi}{d y}=\frac{\alpha(\alpha \hat{u}-\omega)}{\mathrm{R} \overline{\mathrm{T}}}-\frac{\chi^{2} G+\chi d \bar{u} / d y}{(\bar{u}-\omega / \alpha)}, \\
G=\frac{\alpha(\alpha \hat{u}-\omega)}{\mathrm{R} \overline{\mathrm{T}}}-\frac{\chi^{2} G+\chi d \bar{u} / d y}{(\bar{u}-\omega / \alpha)},
\end{gathered}
$$

where $p$ and $v$ are the pressure and normal velocity eigenfunctions, $M a$ is the Mach number of the inner jet $\bar{u}, \bar{\rho}$ and $\bar{T}$ are the base flow streamwise velocity component, density and temperature, $\gamma$ is the specific heat ratio and $R$ is the mixture gas constant.

As described by Sandham (1990), the boundary conditions are given by

$$
\chi(y \rightarrow+/-\infty)=\frac{\alpha \bar{u}-\omega}{\sqrt{G R \bar{T}}} .
$$

The problem posed by the Rayleigh equation, written in terms of the Gropengiesser variable and the corresponding boundary conditions is an eigenvalue problem in terms of $\alpha, \beta, \omega, M a$ and $R_{\text {ratio. The }}$ eigenvalue problem is solved using a classic shooting method for ordinary differential equations (Drazin and Reid, 1981).

\section{Base Flow}

The present investigation considers simple planar jets and co-flowing planar jets. For the coflowing planar jet, an inner jet is surrounded by an outer jet symmetric with respect to the inner jet center line above and below.

The base flow appears in the equations due to decomposition of the flow variables into a base component plus a small oscillation. The present work uses the same base flow velocity profile as Weder (2012) which was also studied by Michalke (1984):

$$
\bar{u}(y)=\lim _{\varsigma \rightarrow y} \frac{1}{2}\left[1+\tanh \frac{R_{1 / 2}}{4 \theta}\left(\frac{R_{1 / 2}}{|\varsigma|}-\frac{|\zeta|}{R_{1 / 2}}\right)\right]
$$

where $R_{1 / 2}$ is the jet half width and $\theta$ is the momentum boundary layer thickness.

For the co-flowing jet the profile is also based on the above equation, but an outer stream is considered. The outer stream has speed equal to 0.7 of the inner jet.

The jet is assumed to be at a constant temperature and variations are only due to viscous dissipation. For the temperature profile the CroccoBusemann relation is used. This relation is preferred to hyperbolic approximations as mentioned by Mendonca (2014). The pressure across the jet is assumed uniform. The chemical species at the inner jet is different from the surrounding ambient for simple jets. For the co-flowing jets the inner and outer jets are streams of different chemical species and the outer jet is of the same chemical species as the ambient environment.

Considering the inner and outer jet streams at the same temperature and pressure of the quiescent ambient, through the equation of state a relation between the base flow gas constant ratio $R_{\text {ratio }}$ and the density ratio $\rho_{\text {ratio }}$ is $R_{\text {ratio }}=1 / \rho_{\text {ratio }}$. Considering the inner jet as the reference state, $R_{\text {ratio }}>1$ corresponds to $\rho_{\text {ratio }}<1$, which corresponds to a heavier jet at the inner stream and $R_{\text {ratio }}<1$ corresponds to $\rho_{\text {ratio }}>1$ with lighter jet at the inner stream.

\section{Numerical Method}

Since the stability equation does not have an analytical closed form solution, numerical methods must be used to solve the problem. In this work the equation is solved using a shooting method, where the equation is integrated from the two boundaries to the center at $y=0$ for a given initial guess for the complex wavenumber $\alpha$, and specified frequency $\omega$, spanwise wavenumber $\beta$, Mach number $M a$ and gas constant ratio $R_{\text {ratio. }}$ If the guessed wavenumber is incorrect the solutions coming from above and below 
$y=0$ will not match and the value of $\alpha$ has to be corrected using a root finding algorithm such as the secant method. The maximum error imposed for the root finding secant method was set to $10^{-8}$. Code verification was performed comparing results with Weder (2012).

To integrate the differential equation from the boundaries towards the center a fourth order RungeKutta method is used. The numerical solution of initial value problems posed by ordinary differential equations achieve high resolution with a low computational cost. In order to assess the numerical accuracy of the model the fastest growing mode for the homogeneous simple jet varicose and sinuous modes were computed with four levels of grid refinement, 251, 501, 1001 and 2001 grid points. For the sinuous mode the fastest growing mode frequency is 0.9496 and the corresponding streamwise wavenumber and growth rate are 1.840466 and 0.735639 . The three higher resolution grids result in identical values and the lowest resolution grid gives 1.840463 and 0.735639 . For the varicose mode the fastest growing mode frequency is 0.9616 and the corresponding streamwise wavenumber and growth rate values obtained with all four grid refinement levels were 1.762716 and 0.743172 . As the numerical solution takes very little computational time, a grid with 1001 grid points was adopted.

\section{RESULTS}

To investigate the spatial stability of the simple jet and co-flowing jet configurations, solutions for the dispersion relation $\mathrm{D}\left(R_{\text {ratio }}, M a, \alpha, \omega\right)=0$ are sought. The gas constant ratios considered for both simple and co-flowing jets are $R_{\text {ratio }}=0.125 ; 0.25 ; 0.5 ; 1.0$; 2.0; 4.0 and 8.0. The inner jet Mach number is kept constant and equal to $M a=0.9$. Both sinuous modes and varicose modes are found and characterized by $\hat{p}(0)=0$ and $\hat{v}(0)=0$ respectively. Results are presented in terms of phase speeds $\mathrm{cp}=\omega / \alpha_{\mathrm{r}}$, , growth rates $\alpha_{\mathrm{i}}$ and flow structure given by $\hat{p}(y)$.

\section{Simple Jet}

This section investigates the spatial stability of a single stream, plane jet flow. The growth rates corresponding to the fastest growing modes are numerically given in Tab. 1 for each gas ratio $R_{\text {ratio, }}$, along with the corresponding angular frequency, streamwise wavenumber and phase speed. For both the sinuous and varicose modes the frequency and phase speed corresponding to the fastest growing mode increase continuously with increasing gas ratio.

\section{Simple Jet Sinuous Mode}

Figure 2 shows growth rate and phase speed, as a function of the angular frequency for different gas ratios for the sinuous case. Comparisons with results from Weder (2012) considering gas ratio equal to 1 and Mach number 0.9 are also presented and serve as validation for the stability code used in this investigation. The comparison with the results shows a very good agreement between both works.

Table 1. Largest spatial growth rate and corresponding angular frequency, wavenumber and phase speed for the simple jet.

\begin{tabular}{ccrcc}
$R_{\text {ratio }}$ & $\omega$ & $\alpha_{\mathrm{r}}$ & $\alpha_{\mathrm{i}}$ & $c p$ \\
\hline \multicolumn{5}{c}{ Sinuous } \\
\hline 0.125 & 0.313 & 1.231 & 0.692 & 0.254 \\
0.250 & 0.483 & 1.457 & 0.742 & 0.332 \\
0.500 & 0.670 & 1.666 & 0.761 & 0.420 \\
1.000 & 0.949 & 1.840 & 0.736 & 0.516 \\
2.000 & 1.202 & 1.954 & 0.660 & 0.615 \\
4.000 & 1.418 & 1.997 & 0.546 & 0.709 \\
8.000 & 1.547 & 1.959 & 0.416 & 0.789 \\
\hline \multicolumn{5}{c}{ Varicose } \\
0.125 & 0.360 & 1.124 & 0.616 & 0.320 \\
0.250 & 0.516 & 1.349 & 0.731 & 0.383 \\
0.500 & 0.717 & 1.565 & 0.770 & 0.458 \\
1.000 & 0.962 & 1.763 & 0.743 & 0.546 \\
2.000 & 1.230 & 1.927 & 0.659 & 0.638 \\
4.000 & 1.488 & 2.050 & 0.532 & 0.726 \\
8.000 & 1.690 & 2.110 & 0.391 & 0.801
\end{tabular}
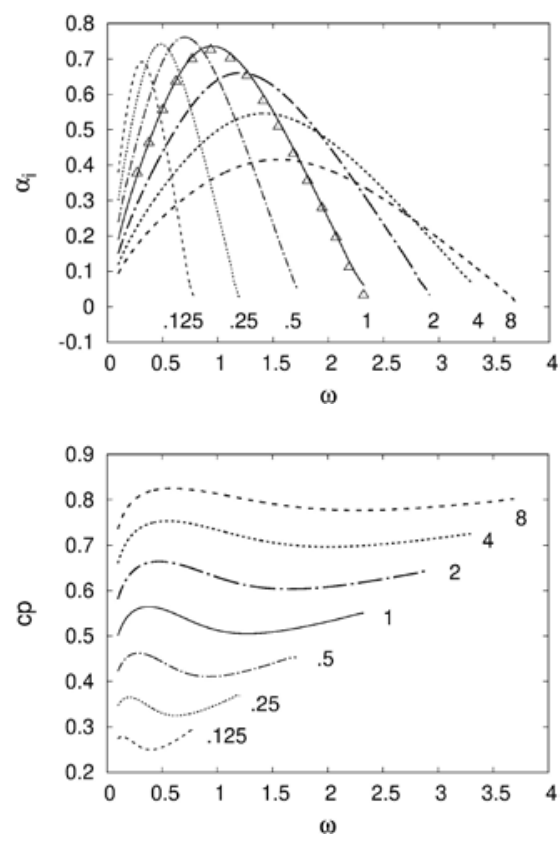

Figure 2. Sinuous mode spatial amplification rate and phase velocity versus angular frequency for different $R_{\text {ratio. }}$ Simple jet.

For gas ratios from 0.125 to 1.0 the largest amplification rate does not to have significant variations, with the highest value at $R_{\text {ratio }}=0.5$. For gas ratios from 2 to 8 the maximum amplification 
rate decreases significantly. Besides the fact that the maximum amplification rate is lower at higher gas ratios, the range of frequencies in which the flow can become unstable is wider than those of lower gas ratios. The phase speeds show that increasing the gas ratio results in less dispersive disturbances as well as in higher phase speeds. The phase speed drops from around $80 \%$ of the jet centerline velocity for $R_{\text {ratio }}=8$ to less than $30 \%$ for $R_{\text {ratio }}=0.125$. Given that the density ratio is proportional to the inverse of the gas constant ratio, the disturbances travel much slower when the lighter fluid is in the jet and the heavier fluid is the ambient fluid.

Figure 3 show the real and imaginary parts of the eigenfunctions $\hat{p}(y)$ for two values of $R_{\text {ratio. The }}$ jet profile is also presented and shows the strong correlation between the shear layer at the jet border and the eigenfunction maximum amplitudes.
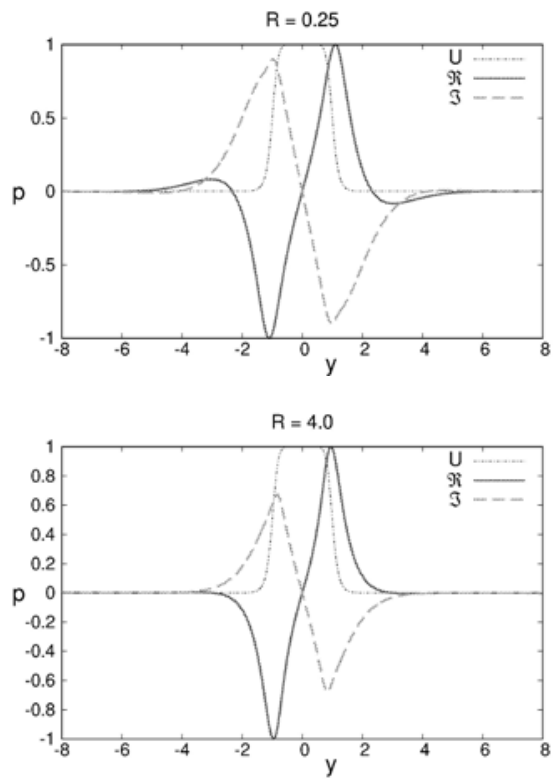

Figure 3. Pressure $\hat{p}(y)$ real and imaginary parts for the sinuous mode largest spatial growth rate for different $R_{\text {ratio }}$. Simple jet.

\section{Simple Jet Varicose Mode}

Results are now presented for the varicose case. Figure 4 shows the amplification rate and the phase speed as a function of angular frequency. The behavior of the varicose instability is very similar to the sinuous. In the varicose case below $R_{\text {ratio }}=1$ the largest amplification rate decay faster than in the sinuous case, but the range of unstable frequencies do not change significantly from the sinuous to the varicose mode. The range of unstable frequencies increases with increasing gas ratio. The phase speed distribution shows that the disturbances are much more dispersive than the sinuous disturbances. The lower the gas ratio, the more dispersive are the disturbances. The phase speed is also strongly

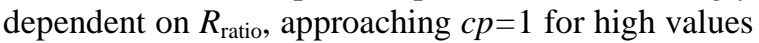

of gas ratio, and 0.3 for the lowest value of the gas ratio. For $R_{\text {ratio }}=1$ growth rates and streamwise wavenumber are compared to the results presented by Weder (2012) showing very good agreement.
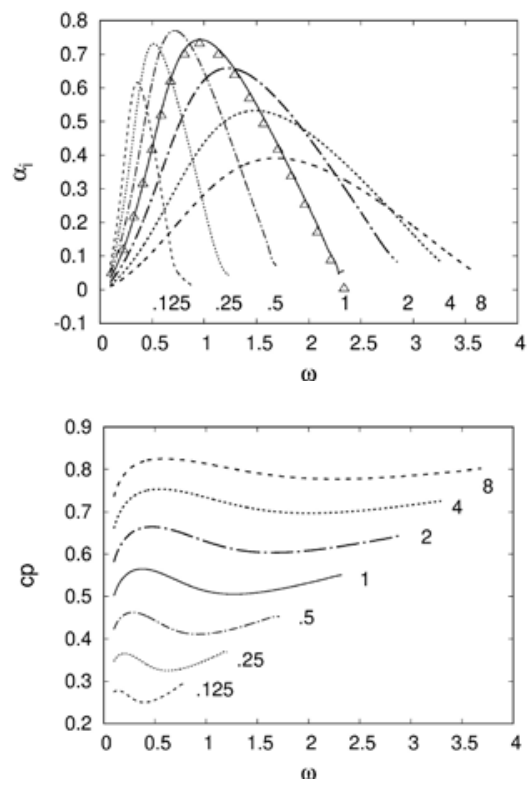

Figure 4. Varicose mode spatial amplification rate and phase velocity versus angular frequency for different $R_{\text {ratio }}$ Simple jet.

The flow structure is presented by the eigenfunctions for pressure real and imaginary parts as shown if Fig. 5 for $R_{\text {ratio }}$ equal to 0.25 and 4 . These results correspond to the frequencies of the largest amplification rate for each $R_{\text {ratio. In this case, no }}$ significant change in phase due to changes in the gas ratio is observed.
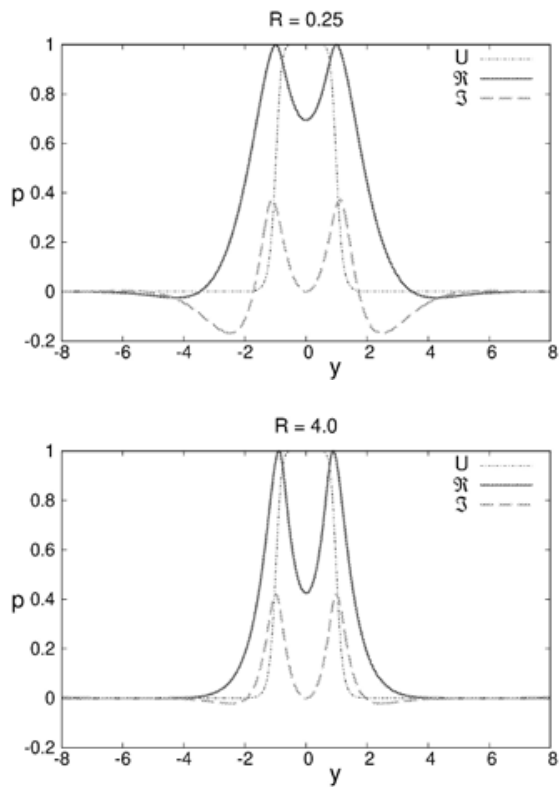

Figure 5. Pressure $\hat{p}(y)$ real and imaginary parts for the varicose mode largest spatial growth rate for different $R_{\text {ratio }}$. Simple jet. 


\section{Co-flowing Jet}

This section investigates the spatial stability of the co-flowing jet flow. The distribution of gas species considers the outer jet and the ambient composed of the same chemical species and a different species at the inner jet.

Since the co-flowing jet has two shear layers, one between the inner jet and the outer jet and one between the outer jet and the ambient, two different modes where found, an internal mode and an external mode. Both internal and external modes have sinuous and varicose instability modes.

The largest growth rates, for each instability mode for each gas ratio and the corresponding angular frequency, streamwise wavenumber and phase speed are given in Tabs. 2 and 3 for the sinuous and varicose modes, respectively. While the growth rate for the internal mode is largest for $R_{\text {ratio }}=1$, the external mode growth rate grows continuously for increasing gas ratio. Comparing both tables, the results show that the sinuous and varicose modes do not differ much in terms of largest growth rates and corresponding angular frequencies for a given gas ratio.

Table 2. Maximum spatial amplification rate and corresponding angular frequency, wavenumber and phase speed for the sinuous mode, co-flowing jet.

\begin{tabular}{cccccc} 
Sinuous & $R_{\text {ratio }}$ & $\omega$ & $\alpha_{\mathrm{r}}$ & $\alpha_{\mathrm{i}}$ & $c p$ \\
\hline & 0.125 & 1.027 & 1.324 & 0.067 & 0.775 \\
& 0.250 & 1.138 & 1.427 & 0.087 & 0.798 \\
Internal & 1.000 & 1.309 & 1.534 & 0.112 & 0.853 \\
mode & 2.000 & 1.356 & 1.535 & 0.110 & 0.883 \\
& 4.000 & 1.364 & 1.498 & 0.099 & 0.911 \\
& 8.000 & 1.328 & 1.422 & 0.082 & 0.934 \\
\hline \multirow{5}{*}{ External } & 0.125 & 0.248 & 0.701 & 0.225 & 0.354 \\
mode & 1.000 & 0.473 & 1.293 & 0.630 & 0.366 \\
& 0.250 & 0.403 & 1.121 & 0.413 & 0.359 \\
& 4.000 & 0.474 & 1.285 & 0.678 & 0.369 \\
& 8.000 & 0.473 & 1.274 & 0.704 & 0.371 \\
\hline
\end{tabular}

The growth rates of the external mode are much higher than the growth rates of the internal modes. The external modes have growth rates comparable to the growth rates of the simple jet presented in Tab. 1, although the co-flowing jet frequencies do not depend on the gas ratio as much as the frequencies of the simple jet. This has significant impact on the mixture of different species, since the interface between different species at the boundary of the inner and the outer jets is more stable. The larger growth rates of the external mode are due to the choice of velocity ratio between the inner jet and the outer jet equal to 0.7 , such that the external velocity gradient is higher.

Table 3. Maximum spatial amplification rate and corresponding angular frequency, wavenumber and phase speed for the varicose mode, co-flowing jet.

\begin{tabular}{cccccc} 
Varicose & $R_{\text {ratio }}$ & $\omega$ & $\alpha_{\mathrm{r}}$ & $\alpha_{\mathrm{i}}$ & $c p$ \\
\hline & 0.125 & 0.950 & 1.207 & 0.062 & 0.787 \\
& 0.250 & 1.086 & 1.340 & 0.082 & 0.810 \\
Internal & 1.000 & 1.353 & 1.560 & 0.103 & 0.867 \\
mode & 2.000 & 1.477 & 1.648 & 0.097 & 0.896 \\
& 4.000 & 1.576 & 1.708 & 0.083 & 0.923 \\
& 8.000 & 1.627 & 1.723 & 0.063 & 0.944 \\
\hline \multirow{5}{*}{ External } & 0.125 & 0.261 & 0.716 & 0.238 & 0.364 \\
mode & 1.000 & 0.470 & 1.279 & 0.631 & 0.367 \\
& 0.250 & 0.400 & 1.107 & 0.414 & 0.361 \\
& 4.000 & 0.472 & 1.275 & 0.679 & 0.370 \\
& 8.000 & 0.471 & 1.268 & 0.704 & 0.371 \\
\hline
\end{tabular}

\section{Co-flowing Jet Sinuous Mode}

Figure 6 shows the spatial amplification rate as a function of angular frequency for the external and internal modes for the sinuous instability, respectively. For the external mode, lowering the gas ratio $R_{\text {ratio }}$ has a stabilizing effect, as the spatial amplification rate and range of frequency are reduced considerably. It is interesting that changing the gas ratio between the inner and outer jets changes the growth rate at the interface between the outer gas and the ambient which are composed of the same species. The stability curves for the external modes are truncated because of convergence problems at higher angular frequencies for high gas ratios.

For the internal jet, the growth rate variation with gas ratio is not monotonic and varies by $50 \%$ between the largest growth rate and the smaller, with the largest growth rate around $R_{\text {ratio }}=1$. The growth rates are much lower that the growth rates of the external modes. For $R_{\text {ratio }}>1$ frequency range increases and the maximum amplification decreases.

Figure 7 shows the phase speed variation with angular frequency and gas ratio. The disturbances are less dispersive for low gas ratios when the external mode is considered, but less dispersive for high gas ratios when the internal mode is considered. The former is more dispersive at high frequencies and the latter is more dispersive at low frequencies. In a white noise environment these results may have an impact on the resulting flow structure and celerity in the transition to turbulence, but a conclusive analysis would depend on the non-linear regime. 

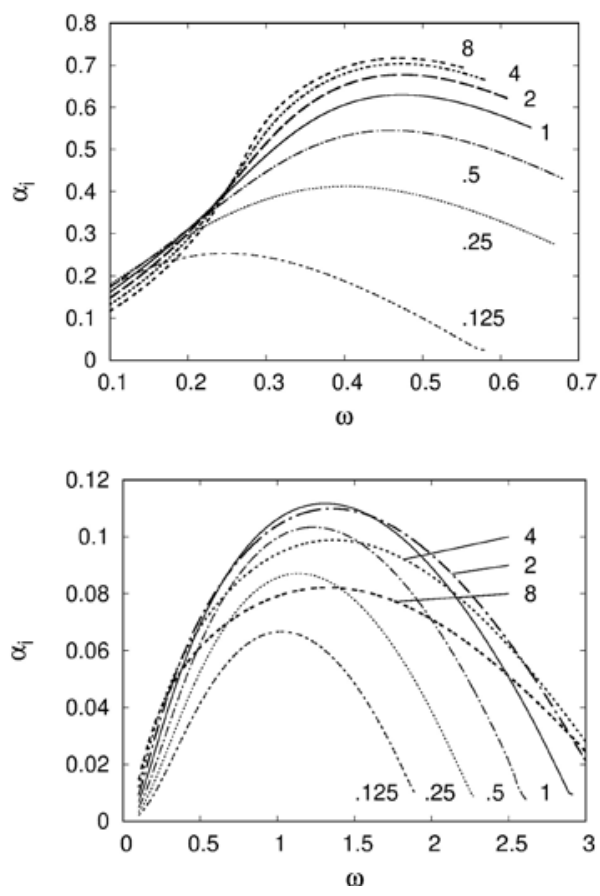

Figure 6. Spatial amplification rate, sinuous mode versus angular frequency, different $R_{\text {ratio }}$, external and internal modes. Co-flowing jet.
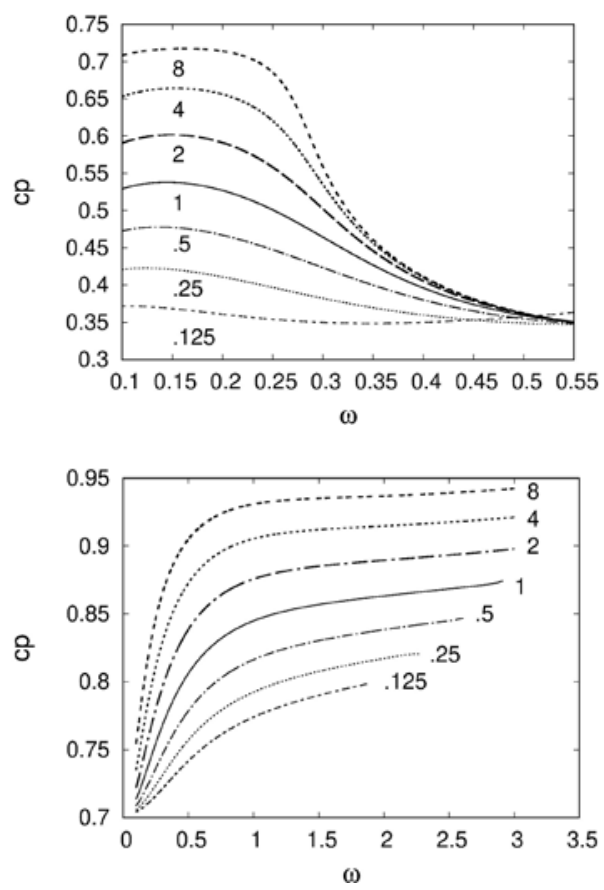

Figure 7. Phase velocity for the sinuous mode versus angular frequency, different $R_{\text {ratio, external and }}$ internal modes. Co-flowing jet.

The pressure real and imaginary eigenfunctions are presented in Figs. 8 and 9 for the outer and inner mode, for the frequency with the largest growth rate in each case. The correlation between the eigenfunction and the shear layer allows the identification of the inner and outer modes. Note that for $R_{\text {ratio }}=0.25$ in Fig. 9 a radiating mode was found, indicating that this is an acoustic mode that decay slowly, oscillating away from the shear layer. For the gas ratio equal to $R_{\text {ratio }}=4$ an exponentially decaying mode was found.
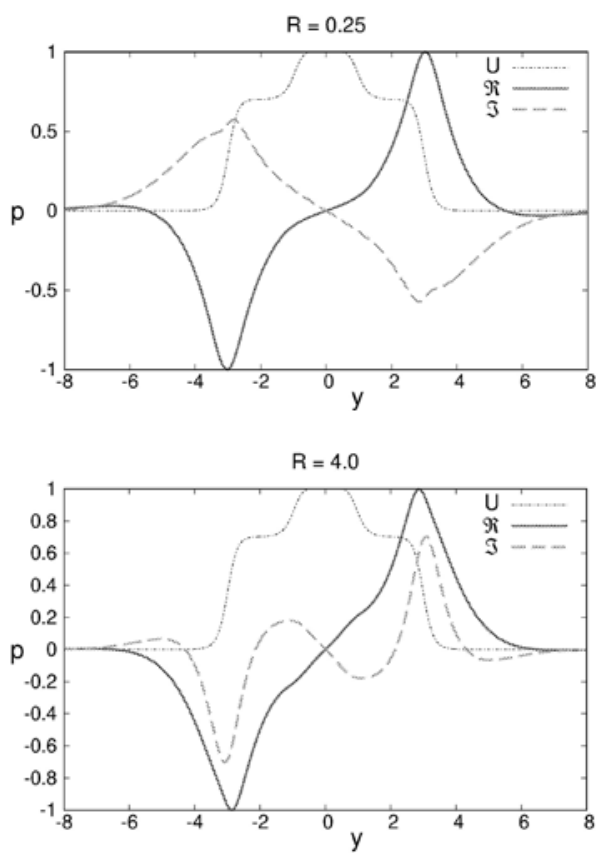

Figure 8. Pressure $\hat{p}(y)$ real and imaginary parts for the sinuous mode for different gas ratios, external mode. Co-flowing jet.
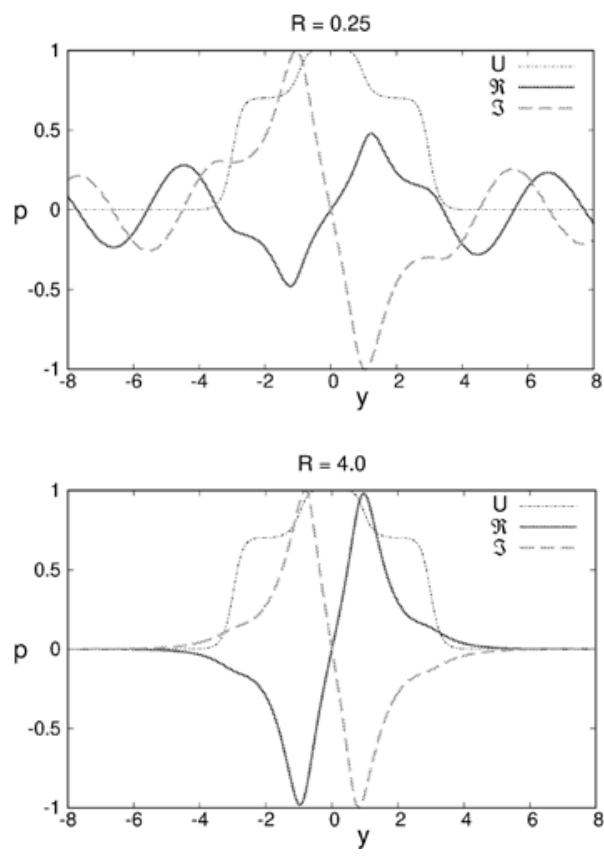

Figure 9. Pressure $\hat{p}(y)$ real and imaginary parts for the sinuous mode for different gas ratios, internal mode. Co-flowing jet.

\section{Co-flowing Jet Varicose Mode}

For the co-flowing jet varicose mode, Figs. 10 
and 11 show the growth rate and phase speed as a function of the angular frequency for a range of gas ratios, both for the internal and external modes. In terms of growth rate and streamwise wave number, the differences between the varicose and sinuous mode seems to be mild and follow the same trend. The distribution of phase speed shows that the varicose, internal mode is much less dispersive in the low frequency limit, while the external mode is somewhat more dispersive. The external mode is less dispersive only for the high frequency range.
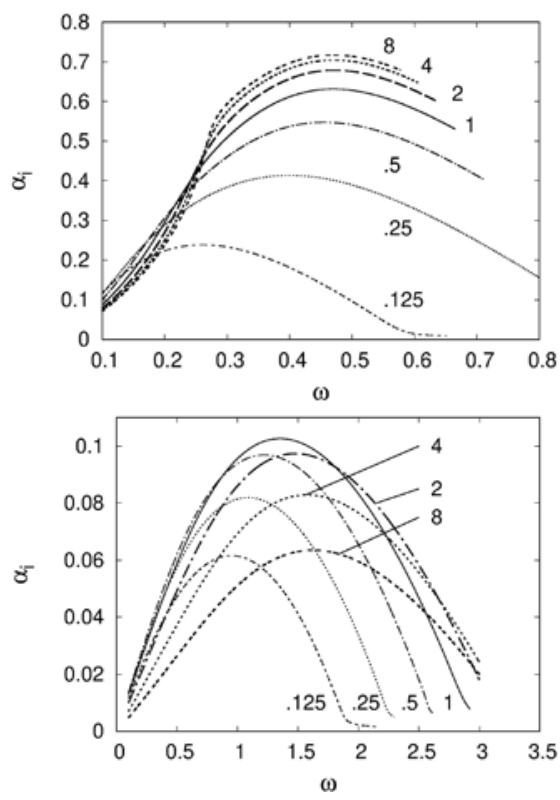

Figure 10. Varicose mode spatial amplification rate versus angular frequency, for different gas ratios, external and internal modes. Co-flowing jet.
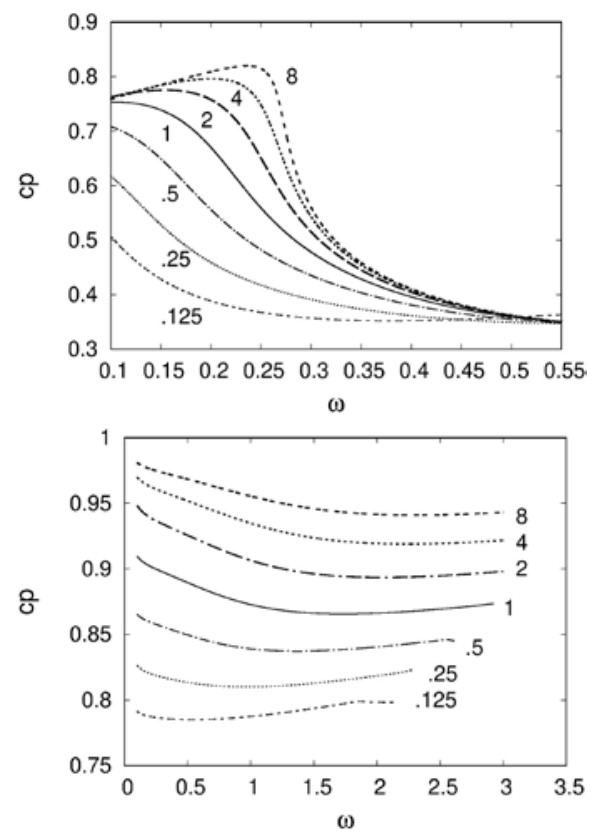

Figure 11. Varicose mode phase velocity versus angular frequency for different gas ratios, external and internal modes. Co-flowing jet.
The effect of binary mixing is similar to the effect found for the sinuous mode, the external mode is strongly damped with decreasing gas ratio, both in terms of growth rate and range of unstable frequencies. The internal mode is less sensitive to the effect of binary mixing, where the largest amplification rate varies by about $50 \%$ and the range of unstable frequencies is reduced by the same amount for the lower values of gas ratio.

The flow structure for the varicose mode eigenfunctions are presented in Fig. 12 and 13. As in the sinuous mode, a radiating mode was found for the varicose, internal mode.
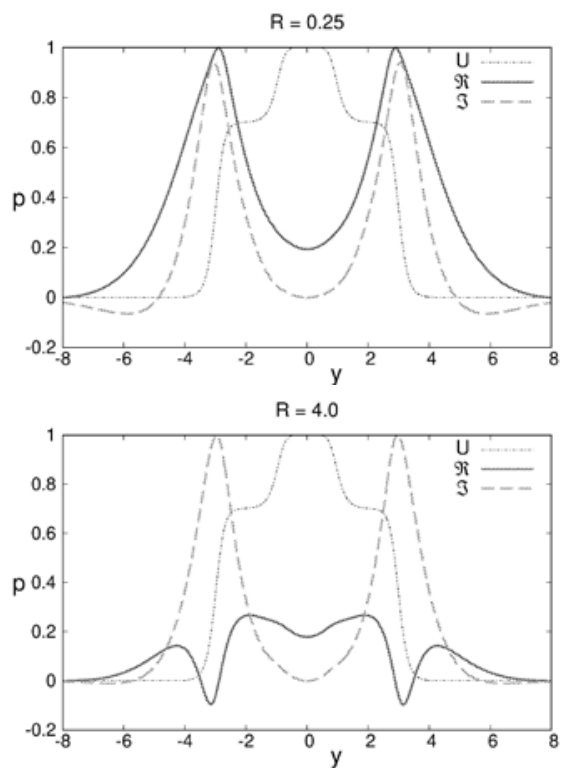

Figure 12. Pressure $\hat{p}(y)$ real and imaginary parts varicose mode for different gas ratios, external mode. Co-flowing jet.
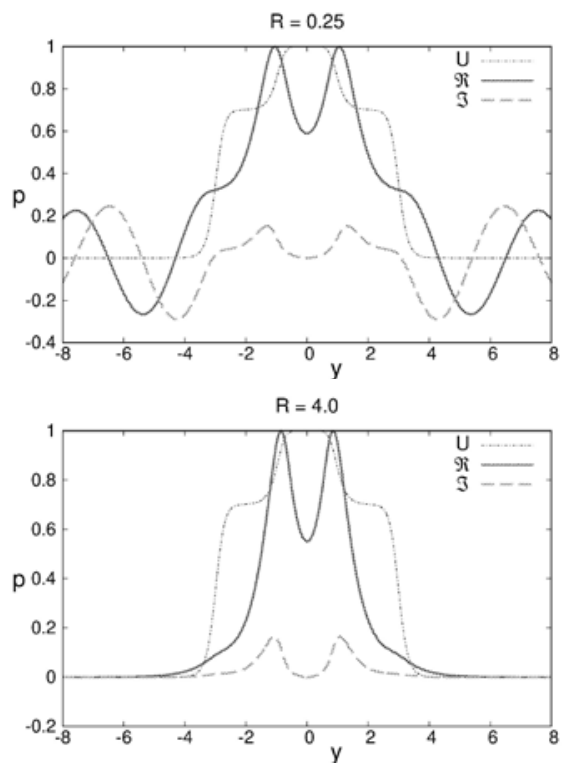

Figure 13. Pressure $\hat{p}(y)$ real and imaginary parts varicose mode for different gas ratios, internal mode. Co-flowing jet. 


\section{CONCLUSIONS}

The present investigation addressed the issue of free shear layers composed of simple and co-flowing jets with different chemical species in the different streams. Different density ratios where considered and the effect of density ratio on the growth rate, range of unstable frequencies and disturbances phase speeds were studied. The resulting flow structures were also considered through the pressure disturbances. For the simple jet, density stratification has a stabilizing effect, but the varicose mode is more dispersive when the jet density is higher than the quiescent ambient. For the co-flowing jet configuration, four different instability modes were found, a sinuous and a varicose mode for the internal and external modes. The density stratification also has a stabilizing effect, except for the external mode, for which the growth rate increases monotonically with decreasing density ratio. The external mode, both for the varicose and sinuous modes, is more dispersive than the internal mode. The results for the co-flowing jet also show that the varicose and sinuous modes support radiating modes away from the jet. The range of density ratios considered and the results for co-flowing jets extend the information regarding the stability of binary free shear layers available in the literature. Considering the development of both inner and outer modes and the difference in phase speeds of each mode, the results indicate that in a nonlinear regime the interaction between the inner and outer modes may hasten transition to turbulence. An extension of the present work will consider a non-parallel, non-linear model based on the parabolized stability equations (PSE) in order to access nonlinear effects. Further investigation on the characteristics of the radiating modes will also be conducted.

\section{ACKNOWLEDGEMENTS}

The first author acknowledges the fellowship from Conselho Nacional de Desenvolvimento Científico e Tecnológico (CNPq). Project funded by CNPq through grant number PVE-401032/2014-0.

\section{REFERENCES}

Drazin, P. G., and Reid, W. H., 1981, Hydrodynamic Stability, Cambridge University Press.

Fedioun, I. and, Lardjane, N., 2005, Temporal Linear Stability Analysis of Three-Dimensional Compressible Binary Shear Layers, AIAA Journal, Vol. 43, No. 1, pp. 111-123.

Gropengiesser, H., 1970, Study on the Stability of Boundary Layers and Compressible Fluids, Technical Report Report TT F-12786, NASA.

Jackson, T. L., and Grosch, C. E., 1989, Inviscid Spatial Stability of a Compressible Mixing Layer, Journal Fluid Mechanics, Vol. 208, pp. 609-637.

Jackson, T. L., and Grosch, C. E., 1990,
Absolute/Convective Instabilities and the Convective Mach Number in a Compressible Mixing Layer, Physics of Fluids A, Vol. 2, No. 6, pp. 949-954.

Kennedy, C. A., J. H. Chen, M. Y. H., and Jackson, T. L., 1998. Mean Flow Effects on the Linear Stability of Compressible Planar Jets, Physics of Fluids, Vol. 10, No. 3, pp. 615-626.

Kennedy, C. A., and Gatski, T. B., 1994, SelfSimilar Supersonic Variable Density Shear Layers in Binary Systems, Physics of Fluids A, Vol. 6, No. 2, pp. 662-673.

Kozusko, F., Lasseigne, D. G., Grosch, C. E., and Jackson, T. L., 1996, The Structure of Variable Property Compressible Mixing Layers in Binary Gas Mixtures, Physics of Fluids, Vol. 8, No. 7, pp. 19451953.

Kozusko, F., Lasseigne, D. G., Grosch, C. E., and Jackson, T. L., 1996b, The Stability of Compressible Mixing Layers in Binary Gases, Physics of Fluids, Vol. 8, No. 7, pp. 1954-1963.

Lacerda, J. F., Souza, L. F., Rogenski, J. K., and Mendonca, M. T., 2018, Direct Numerical Simulation Code Validation for Compressible Shear Flows Using Linear Stability Theory, Journal Aerospace Technology and Management, Vol. 10, No. 184, pp. 1-13.

Manco, J. A. A., Freitas, R. B., Fernandes, L. M., and Mendonca, M. T., 2015, Stability of Compressible Mixing Layers Modified by Wakes and Jets, in: Procedia IUTAM, IUTAM Symposium. on Laminar-Turbulent Transition, Rio de Janeiro, Vol. 14, pp. $129-136$.

Mendonca, M. T., 2014, Linear Stability Analysis of Binary Compressible Mixing Layers Modified by a Jet or a Wake Deficit, in: 52nd AIAA Aerospace Sciences Meeting and Exhibit,USA.

Michalke, A., 1984, Survey on Jet Instability Theory, Progress in Aerospace Sciences, Vol. 21, pp. 159-199.

Rogenski, J. K., Souza, L. F., de Mendonca, M. T., and Morris, P. J., 2017, Growth of Even and Odd Instability Modes in Compressible Binary Jet Flows, in: 24th ABCM International. Congress of Mechanical Engineering, COBEM, ABCM, Curitiba, PR.

Salemi, L., and Mendonca, M. T., 2008, Spatial and Temporal Linear Stability Analysis of Binary Compressible Shear Layer, in: AIAA 38th Fluid Dynamics Conference, USA, AIAA 2008-3841.

Sandham, N. D., 1990, A Numerical Investigation of the Compressible Mixing Layer, Doctoral Thesis, Stanfor University, USA.

Tam, C. K. W., and Thies, A. T., 1993, Instability of Rectangular Jets, Journal of Fluid Mechanics, Vol. 248, pp. 425-448.

Weder, M., 2012, Linear Stability and Acoustics of a Subsonic Plane Jet Flow, Master Thesis, Institute of Fluid Dynamics, ETH, Zurich.

Zhuang, M., Kubota, T., and Dimotakis, P. E., 1990, Instability of Inviscid Compressible Free Shear Layers, AIAA Journal, Vol. 28, No. 10, pp. 17281733. 